\title{
Drug treatment for attention-deficit/hyperactivity disorder and suicidal behaviour: register based study
}

\author{
(C) $(1) \Theta$ OPEN ACCESS
}

\author{
Qi Chen PhD student ${ }^{1}$, Arvid Sjölander associate professor ${ }^{1}$, Bo Runeson professor ${ }^{2}$, Brian M \\ D'Onofrio associate professor ${ }^{3}$, Paul Lichtenstein professor $^{1}$, Henrik Larsson associate professor ${ }^{1}$
}

'Department of Medical Epidemiology and Biostatistics, Karolinska Institute, Box 281, 17177 Stockholm, Sweden; ${ }^{2}$ Department of Clinical Neuroscience, Karolinska Institute, Stockholm, Sweden; ${ }^{3}$ Department of Psychological and Brain Sciences, Indiana University, Bloomington, IN, USA

\begin{abstract}
Objective To investigate the association between drug treatment for attention-deficit/hyperactivity disorder (ADHD) and risk of concomitant suicidal behaviour among patients with ADHD.

Design Register based longitudinal study using within patient design.

Setting Linkage of multiple national registers in Sweden.

Participants 37936 patients with ADHD born between 1960 and 1996 and followed from 2006 to 2009 for treatment status by ADHD drug treatment and suicide related events (suicide attempt and completed suicide).
\end{abstract}

Main outcome measure Incidence rate of suicide related events during ADHD drug treatment periods compared with that during non-treatment periods.

Results Among 37936 patients with ADHD, 7019 suicide related events occurred during 150721 person years of follow-up. At the population level, drug treatment of ADHD was associated with an increased rate of suicide related events (hazard ratio 1.31, 95\% confidence interval 1.19 to 1.44). However, the within patient comparison showed a reverse association between ADHD drug treatment and rate of suicide related events $(0.89,0.79$ to 1.00$)$. Among stimulant users, a reduced within patient rate of suicide related events was seen during treatment periods $(0.81,0.70$ to 0.94$)$. Among non-stimulant/mixed users, no significantly increased within patient rate of suicide related events during non-stimulant treatment periods was seen $(0.96,0.72$ to 1.30$)$.

Conclusions This study found no evidence for a positive association between the use of drug treatments for ADHD and the risk of concomitant suicidal behaviour among patients with ADHD. If anything, the results pointed to a potential protective effect of drugs for ADHD on suicidal behaviour, particularly for stimulant drugs. The study highlights the importance of using within patient designs to control for confounding in future pharmacoepidemiological studies.

\section{Introduction}

Attention-deficit/hyperactivity disorder (ADHD) is a common neurodevelopmental disorder that can affect people across the lifespan. ${ }^{1-4}$ The prescribing rates of drug treatments for ADHD have increased substantially in the United States and Europe, ${ }^{5-7}$ probably because of their documented short term efficacy in symptom management and improving social functioning among patients at different ages. ${ }^{8-11}$ However, drug safety as regards rare but serious adverse events, such as suicidal behaviour, has not been comprehensively evaluated.

On the basis of 12 short term placebo controlled clinical trials, the Food and Drug Administration raised a black box warning on an elevated risk of suicidal ideation in children and adolescents being treated with atomoxetine, a non-stimulant drug for the treatment of ADHD. ${ }^{12}$ The warning recommended families and caregivers to closely monitor patients with ADHD not only for suicidal ideation but also for suicidal behaviour, especially during the initial stage of treatment or at times of changing doses. A meta-analysis of 14 clinical trials also reported a statistically significant association between use of atomoxetine and suicidal ideation, but not suicidal behaviour. ${ }^{13}$ Results from observational studies suggested an increased risk of completed suicide among drug treated ADHD patients aged 11 to 14 , compared with the general population, ${ }^{14}$ and also similar rates of suicide related events in patients receiving atomoxetine compared with those receiving methylphenidate, the most commonly prescribed stimulant for ADHD treatment. ${ }^{15}$ The rarity of suicide related events (completed suicide or suicide attempt) in previous studies leads to substantial uncertainty in the interpretation of the results. Whether the possible treatment emergent suicidal ideation suggested by the randomised controlled trials may vanish as the treatment proceeds or may eventually develop into suicidal behaviour is unknown.

Furthermore, the effect of methylphenidate and other stimulant drugs on suicidal behaviour remains unclear.

Drug safety assessment for rare adverse events is largely hindered by methodological challenges. Randomised controlled trials are often based on small sample sizes, short durations of follow-up, and strict inclusion and exclusion criteria, which 
constrains the ability to explore drug safety, especially for rare events. ${ }^{16-18}$ Observational studies may offer large sample sizes with adequate numbers of rare events and long term follow-up, but insufficient adjustment for confounding might result in biased conclusions in such studies. For instance, ADHD drug treatment may be only an indicator of the disorder or its comorbid conditions, which themselves might increase susceptibility for suicidal behaviour, ${ }^{19}$ so attributing the risk of suicidal behaviour simply to ADHD drug treatment may be unreasonable.

For some patients, the baseline severity of the disorder, along with other characteristics such as familial susceptibility and personal lifestyle, has a time fixed effect throughout the observational period. Confounding by these time fixed factors can be eliminated by within patient comparison analysis whereby each patient serves as his or her own matched control, regardless of whether these factors can be measured. ${ }^{20}$ In this study, we aimed to investigate the association between use of ADHD drug treatment and risk of concomitant suicidal behaviour both at the population (between patient) level and the within patient level. The within patient comparisons implicitly control for unmeasured time fixed confounding before and during the follow-up.

\section{Methods}

\section{Study participants}

The study participants came from the Swedish national patient register, which has complete nationwide coverage of information on psychiatric inpatient care since 1987 and outpatient visits to specialists (non-general practitioners) since 2001; each visit had one primary diagnosis and up to eight secondary diagnoses based on ICD-9 (international classification of diseases, 9th revision) for 1987-96 and ICD-10 since 1997. We identified 38 056 patients born between 1960 and 1996 with a clinical diagnosis of ADHD (ICD-9: 314; ICD-10: F90). We excluded patients who died $(n=81)$ or moved out of Sweden $(n=39)$ before 1 January 2006, leaving a sample of 37936 patients who we followed for their treatment status by ADHD drugs and suicide related events from 1 January 2006 until death, emigration, or 31 December 2009, whichever occurred first. To protect patients' privacy, only anonymous health data were provided by the Swedish government agency, Statistics Sweden, to ensure that no data can be traced back to individual patients.

\section{Treatment status by ADHD drugs}

We ascertained ADHD drug treatment status by dispensed prescriptions recorded in the prescribed drug register. ${ }^{21}$ Specifically, for each patient, we divided the entire follow-up into treatment periods and non-treatment periods. As in previous studies, ${ }^{22}{ }^{23}$ we defined a treatment period as a sequence of prescriptions for ADHD drug treatments with less than six months (183 days) between two consecutive prescriptions; we considered gap of six months or more between prescriptions to indicate a non-treatment period. The start of a treatment period was the date of the first prescription, and the end of the treatment period was the date of the last prescription. We took into consideration prescriptions in 2005 and 2010 to ascertain the treatment status over the first and the last period during the follow-up from 2006 to 2009. The Swedish prescribed drug register provides information on dates of prescriptions and anatomical therapeutic chemical classification codes for all ADHD drugs dispensed to the entire population in Sweden since July $2005 .{ }^{21}$ In this study, we focused on three stimulants (methylphenidate (N06BA04), amfetamine (N06BA01), and dexamfetamine (N06BA02)) and one non-stimulant (atomoxetine (N06BA09)). In our sample, 26150 (68.9\%) patients received ADHD drug prescription at some point between 1 July 2005 and 30 June 2010. Among these treated patients, $24555(93.9 \%)$ received at least one prescription for methylphenidate and $6818(26.1 \%)$ received at least one prescription for atomoxetine. The other two stimulants, amfetamine and dexamfetamine, were rarely prescribed.

\section{Suicide related events}

We defined a suicide related event as a suicide attempt or registered death due to suicide, based on ICD-10 codes (X60-X84: intentional self-harm; Y10-Y34: event of undetermined intent). Dates and diagnoses of suicide attempts came from the national patient register; dates and causes of completed suicides came from the cause of death register. All the suicide related events had been well validated. ${ }^{24}{ }^{25}$

\section{Comorbid conditions}

The Swedish national patient register also provides information on several comorbid conditions related to ADHD or suicidal behaviour, including depressive disorder (ICD-8: 296.2, 298.0, 300.4; ICD-9: 296.2, 296.3, 300.4, 311; ICD-10: F32-F34), bipolar affective disorder (ICD-8: 296.1, 296.3, 296.8; ICD-9: 296.0, 296.4-296.8; ICD-10: F30, F31), conduct disorder (ICD-9: 312; ICD-10: F91), drug abuse (ICD-8: 304; ICD-9: 292, 304; ICD-10: F11-16, F18, F19), and borderline personality disorder (ICD-9: 301.83; ICD-10: F60.3).

\section{Statistical analyses}

We compared the demographic and clinical characteristics between ADHD patients with and without suicide related events, as well as ADHD patients with different treatment patterns (non-treatment, intermittent treatment, and continuous treatment throughout the follow-up). These results formed the basis of the adjustments in the main analyses and the selection of sensitivity analyses.

At the population level, we used Cox regression models to estimate hazard ratios and $95 \%$ confidence intervals for time to suicide related event, with robust standard errors accounting for the correlated data from the same patient. We adjusted these models for sex and categorical age per year. We divided the follow-up time into consecutive periods for each patient. A new period started after a treatment switch (that is, from treatment to non-treatment or vice versa) or a suicide attempt. A period following a treatment switch was left truncated at the time of the switch; a period following a suicide attempt started at baseline (that is, a patient continued contributing time of follow-up after a suicide attempt). A period ending with a treatment switch, emigration, or death due to other causes than suicide was considered censored. A detailed description of the statistical modelling approach can be found in a recent publication. ${ }^{22}$

We used stratified Cox regression models for within patient comparisons, in which time was divided into periods as for the ordinary Cox regression model described above, with each patient as a separate stratum. Stratification on each patient meant that the patient served as his or her own control, so the analyses by design adjusted for all time fixed factors for the same patient before and during the follow-up. The models were adjusted for several time varying covariates, including categorical age per year, previous number of treatment switches, and previous number of suicide attempts. 
We did several sensitivity analyses to examine to what extent our results were affected by variations in the definitions of the exposure and the cohort. We used two additional exposure definitions to examine the potential influence of misclassification of exposure time. Firstly, we set the end of each treatment period to be 30 days after the last prescription. Secondly, we redefined treatment status by using a three month cut-off (that is, the treatment period was defined as a sequence of prescriptions without discontinuation within 92 days).

Because stimulants and non-stimulants differ in terms of pharmacological mechanism and treatment efficacy, ${ }^{26}$ we did analyses in subgroups of stimulant users (patients treated by stimulants only) and non-stimulant/mixed users (patients with at least one treatment period by non-stimulant). In the second subgroup, we further divided the treatment periods into stimulant treatment periods and non-stimulant treatment periods. We respectively compared the rate of suicide related events during these two types of treatment periods with that during non-treatment periods.

Impulsivity might be an important underlying trait of suicidal behaviour, ${ }^{27}$ and the hyperactive/impulsive symptoms of ADHD usually decline over time at a higher rate than do the inattentive symptoms. ${ }^{28}$ Considering the potential age dependent effect of ADHD drug treatment on suicidal behaviour, we studied the associations in a younger cohort born during 1982-96 (baseline age 10-24 years) and an older cohort born during 1960-81 (baseline age 25-46 years).

To examine confounding by comorbid conditions, we first restricted analyses to ADHD patients without comorbid conditions. We then did analyses in ADHD patients with a lifetime diagnosis of depressive disorder, both with and without adjustment for the use of antidepressants. We defined treatment by antidepressants as receipt of antidepressant without discontinuation within three months (92 days). We used SAS software version 9.3 for all statistical analyses.

\section{Results}

A total of 7019 suicide related events occurred in 37936 patients with ADHD during 150721 person years of follow-up. Patients with suicidal behaviour were more likely to be female $(\mathrm{P}<0.001)$ and older $(\mathrm{P}<0.001)$, to have received ADHD drug treatment $(\mathrm{P}<0.001)$ and antidepressants $(\mathrm{P}<0.001)$, and to have more comorbid conditions $(\mathrm{P}<0.001)$, compared with those without suicidal behaviour. Tables $1 \Downarrow$ and $2 \Downarrow$ show the baseline characteristics of ADHD patients with and without suicide related events and according to treatment patterns during follow-up. Among patients exposed to at least one treatment period (that is, patients who received at least two prescriptions within a six month period), $90.3 \%$ also had non-treatment period because they started drug treatment after the beginning of follow-up or discontinued their treatment at least once during follow-up.

\section{ADHD drug treatment and rate of suicide related events}

At the population level, the rate of suicide related events was higher during treatment periods than during non-treatment periods (hazard ratio $1.31,95 \%$ confidence interval 1.19 to 1.44). The increased rate of suicide related events was observed in both sexes (table $3 \Downarrow$ ).

In contrast, when comparing the rates of suicide related events for the same patients over different periods, we found no evidence for an increased rate during treatment periods (hazard ratio $0.89,0.79$ to 1.00 ); rather, we saw an indication of a reduction in the rate of suicide related events during treatment periods, although the $95 \%$ confidence interval included 1.00 . We obtained similar results in both sexes (table $3 \Downarrow$ ).

\section{Sensitivity analyses}

We did not observe a significantly increased within patient rate of suicide related events associated with the use of ADHD drug treatment either by resetting the end of each treatment period or by using a three month cut-off to redefine treatment status. These results suggest that the main results are not due to our definition of treatment status (table $4 \Downarrow$ ).

At the population level, the use of ADHD drug treatment was associated with an increased rate of suicide related events among non-stimulant/mixed users (hazard ratio $1.49,1.27$ to 1.76 ), but the association was not significant among stimulant users (1.02, 0.90 to 1.16 ). Among non-stimulant/mixed users, more suicide related events occurred during non-stimulant treatment periods (hazard ratio $1.48,1.17$ to 1.88 ) than during non-treatment periods (table $4 \Downarrow$ ). In the within patient comparisons, however, no increased rate of suicide related events was associated with the use of ADHD drug treatment regardless of the type of drug. We even found a statistically significant protective effect of stimulants on suicidal behaviour (hazard ratio $0.81,0.70$ to 0.94 ) (table $4 \Downarrow$ ).

Use of ADHD drug treatment seemed to increase the rate of suicide related events at the population level in both the younger cohort (hazard ratio 1.17, 1.05 to 1.31) and the older cohort $(1.39,1.20$ to 1.61$)$, whereas the within patient comparisons showed no increased rate. In the older cohort, a significant reduction in the rate was suggested by the within patient comparison (hazard ratio $0.82,0.68$ to 0.99 ) (table $4 \Downarrow$ ).

After the exclusion of patients with a lifetime diagnosis of any comorbid condition (depressive disorder, bipolar affective disorder, conduct disorder, drug abuse, and borderline personality disorder), only 946 suicide related events remained for the analyses, resulting in wide $95 \%$ confidence intervals (table $4 \Downarrow$ ). The association between the rate of suicide related events and the use of ADHD drug treatment at the population level (hazard ratio $1.24,1.03$ to 1.48 ) was largely attenuated and no longer statistically significant in the within patient comparison $(1.08,0.69$ to 1.70$)$ (table $4 \Downarrow)$. Among patients with a lifetime diagnosis of depressive disorder, the association at the population level (hazard ratio $1.28,1.13$ to 1.43 ) was reversed in the within patient comparison $(0.78,0.68$ to 0.91$)$. Similar results remained even after adjustment for the use of antidepressants during follow-up (table $4 \Downarrow$ ).

\section{Discussion}

In this register based cohort study, we followed 37936 patients with a diagnosis of ADHD for 150721 person years to study the association between the use of drug treatment for ADHD and the risk of suicidal behaviour. At the population level, the use of ADHD drug treatment seemed to be associated with an increased rate of concomitant suicide related events in patients of both sexes. However, these associations were not evident in within patient comparisons in which the point estimates suggested no evidence for a harmful effect of ADHD drug treatment on suicidal behaviour irrespective of whether the drug was stimulant or non-stimulant. In fact, the results suggested that $\mathrm{ADHD}$ drug treatment was associated with a reduction in concomitant suicide related events. Any protective effect is probably mediated by the improvement of ADHD symptoms, particularly impulsivity. 


\section{Strengths and limitations of study}

To the best of our knowledge, this is the first nationwide longitudinal study investigating ADHD drug treatment in relation to suicidal behaviour. By linking Swedish national registers, we identified a total of 7019 validated suicide related events for analysis. Furthermore, the study used within patient comparisons to control for both measured time varying confounding and unmeasured time fixed confounding during the follow-up. The attenuation of the within patient estimates suggests that the increased rate of suicide related events associated with the use of drug treatment at the population level might be due to unmeasured confounding factors, such as baseline severity of ADHD or familial susceptibility to ADHD, rather than the use of ADHD drug treatment. Nevertheless, although we did not find an increased rate of suicide related events in the within patient analyses of non-stimulant drugs, the upper limit of the $95 \%$ confidence interval of the hazard ratio was estimated to be 1.30 , so an elevated rate of suicide related events associated with the use of ADHD drug treatment cannot be excluded.

Depressive disorder frequently co-occurs with ADHD and represents a major risk factor for suicidal behaviour. ${ }^{29}{ }^{30} \mathrm{In}$ October 2004 the Food and Drug Administration issued a black box warning on an increased risk of suicidality associated with antidepressants in the treatment of major depressive disorder among children and adolescents. ${ }^{31}$ In our sample, a large proportion of the ADHD patients also had a diagnosis of depressive disorder and used antidepressants during the follow-up. We accordingly did sensitivity analyses among ADHD patients with a lifetime diagnosis of depressive disorder while adjusting for the use of antidepressants during the follow-up. In the within patient comparisons, we observed a protective effect of ADHD drug treatment on suicidal behaviour, both before and after adjustment for antidepressant treatment status. However, the effect of drug combinations in real life is more complex. Future research needs to use more stringent definitions of depressive episodes and antidepressant treatment status to disentangle the effect of ADHD drug treatment on suicidal behaviour. On the other hand, other psychotropic drugs such as antidepressants have very different patterns of use, as well as onset and end of effect. Thus, the use of psychotropic or other drugs would be unlikely to have the potential to explain the association between ADHD drug treatment and suicidal behaviour.

The results should be interpreted in the context of several limitations. Firstly, the ascertainment of treatment period was based on a sequence of dispensed prescriptions that might inaccurately reflect the actual consumption of drugs by patients, because other family members or healthcare staff could also pick up the drug. This might give rise to misclassification of exposure time. Such a problem is similar to the potential non-adherence to protocol in clinical trials, which can rarely be overcome by the intention to treat analysis used in most randomised controlled trials. In our study, the within patient estimates, based on different definitions of treatment status, did not invalidate the results from the main analyses, indicating that exposure time misclassification is unlikely to account for the results.

Secondly, confounding by time varying factors, such as sporadic onset of comorbid conditions that are associated with both drug treatment and suicide related events, are not taken into account in within patient comparisons. However, we did stratified analyses in a subgroup of ADHD patients without the main psychiatric comorbidities and observed no increased rate of suicide related events associated with the use of ADHD drug treatment.

The validity of diagnosis of ADHD in adults has been considered controversial. ${ }^{32}{ }^{33}$ Nevertheless, studies have consistently reported that $\mathrm{ADHD}$ is a relatively stable condition that persists from childhood into adulthood in a large number of cases and is associated with impairments in both clinical and psychosocial functioning. ${ }^{34}$ The Swedish prescribed drug register contains mainly patients with severe ADHD who received drug treatment when non-drug interventions alone had failed. Therefore, we consider overdiagnosis of ADHD in adults to be unlikely in this study, even though generalisations of the results to patients with mild to moderate ADHD should be made with caution.

\section{Conclusions and implications for future research}

We found no evidence for an overall increased rate of suicide related events associated with the use of drug treatment for ADHD. If anything, the results pointed to a potential protective effect of ADHD drug treatment on suicidal behaviour, particularly for stimulant drugs. Despite the black box warning on atomoxetine related suicidal ideation, no evidence existed for an increased rate of suicide related events associated with the use of atomoxetine among non-stimulant/mixed users in our study. The study also illustrates the importance of using within patient designs to control for confounding in future pharmacoepidemiological studies.

Contributors: QC was involved in the conception of the study, analysis and interpretation of the data, and writing the manuscript. AS, PL, and $\mathrm{HL}$ were involved in the study design and analysis and interpretation of the data. BR and BMD were involved in study conception and interpretation of the data. All authors were involved in revising the article critically for important intellectual content and final approval of the version to be published. $\mathrm{HL}$ was responsible for study supervision and obtaining funding. $\mathrm{HL}$ is the guarantor.

Funding: This study was funded by the Swedish Council for Working Life and Social Research (2012-1678), the Swedish Research Council (2011-2492; 2013-2280), the Åke Wibergs foundation, the Strategic Research Program in Epidemiology at Karolinska Institutet, and a Swedish Initiative for Research on Microdata in the Social and Medical Sciences (SIMSAM) framework grant (340-2013-5867). The funders had no role in study design, data collection and analysis, decision to publish, or preparation of the manuscript.

Competing interests: All authors have completed the ICMJE uniform disclosure form at www.icmje.org/coi_disclosure.pdf (available on request from the corresponding authors) and declare: no support from any organisation for the submitted work other than those listed above; no financial relationships with any organisations that might have an interest in the submitted work in the previous three years; no other relationships or activities that could appear to have influenced the submitted work.

Ethical approval: This study was approved by the ethical committee of Karolinska Institutet (2005/4:5)

Transparency declaration: The lead author affirms that the manuscript is an honest, accurate, and transparent account of the study being reported; that no important aspects of the study have been omitted; and that any discrepancies from the study as planned (and, if relevant, registered) have been explained.

Data sharing: No additional data available.

1 Biederman J. Attention-deficit/hyperactivity disorder: a selective overview. Biol Psychiatry 2005;57:1215-20.

2 Greydanus DE, Pratt HD, Patel DR. Attention deficit hyperactivity disorder across the lifespan: the child, adolescent, and adult. Dis Mon 2007;53:70-131. 


\section{What is already known on this topic}

Patients with attention-deficit/hyperactivity disorder (ADHD) might be at increased risk of suicidal behaviour, compared with the general population

A meta-analysis showed that atomoxetine, a non-stimulant drug used to treat ADHD, might increase the risk of suicidal ideation in patients with ADHD

\section{What this study adds}

This longitudinal study found no evidence for an overall increased rate of suicide related events associated with the use of stimulant or non-stimulant drug treatment for ADHD

An observed protective effect of stimulant drugs on suicidal behaviour among ADHD patients who received only stimulant drugs needs further investigation

3 Polanczyk G, de Lima MS, Horta BL, Biederman J, Rohde LA. The worldwide prevalence of ADHD: a systematic review and metaregression analysis. Am J Psychiatry 2007;164:942-8.

4 Biederman J, Petty CR, Monuteaux MC, Fried R, Byrne D, Mirto T, et al. Adult psychiatric outcomes of girls with attention deficit hyperactivity disorder: 11-year follow-up in a longitudinal case-control study. Am J Psychiatry 2010;167:409-17.

5 Zuvekas SH, Vitiello B. Stimulant medication use in children: a 12-year perspective. Am J Psychiatry 2012;169:160-6.

6 Hodgkins P, Sasane R, Meijer WM. Pharmacologic treatment of attention-deficit/hyperactivity disorder in children: incidence, prevalence, and treatment patterns in the Netherlands. Clin Ther 2011;33:188-203.

7 Zetterqvist J, Asherson P, Halldner L, Langstrom N, Larsson H. Stimulant and non-stimulant attention deficit/hyperactivity disorder drug use: total population study of trends and discontinuation patterns 2006-2009. Acta Psychiatr Scand 2013;128:70-7.

8 Findling RL. Evolution of the treatment of attention-deficit/hyperactivity disorder in children: a review. Clin Ther 2008:30:942-57.

9 Greenhill L, Kollins S, Abikoff H, McCracken J, Riddle M, Swanson J, et al. Efficacy and safety of immediate-release methylphenidate treatment for preschoolers with ADHD. $J$ Am Acad Child Adolesc Psychiatry 2006;45:1284-93.

10 Surman $\mathrm{CB}$, Hammerness PG, Pion K, Faraone SV. Do stimulants improve functioning in adults with ADHD? A review of the literature. Eur Neuropsychopharmacol 2013;23:528-33.

11 Durell TM, Adler LA, Williams DW, Deldar A, McGough JJ, Glaser PE, et al. Atomoxetine treatment of attention-deficit/hyperactivity disorder in young adults with assessment of functional outcomes: a randomized, double-blind, placebo-controlled clinical trial. J Clin Psychopharmacol 2013;33:45-54.

12 Food and Drug Administration. Public health advisory: suicidal thinking in children and adolescents being treated with Strattera (atomoxetine). FDA, 2005

13 Bangs ME, Tauscher-Wisniewski S, Polzer J, Zhang S, Acharya N, Desaiah D, et al. Meta-analysis of suicide-related behavior events in patients treated with atomoxetine. $J$ Am Acad Child Adolesc Psychiatry 2008;47:209-18.

14 McCarthy S, Cranswick N, Potts L, Taylor E, Wong IC. Mortality associated with attention-deficit hyperactivity disorder (ADHD) drug treatment: a retrospective cohort study of children, adolescents and young adults using the general practice research database. Drug Saf 2009;32:1089-96.

15 Bushe CJ, Savill NC. Systematic review of atomoxetine data in childhood and adolescent attention-deficit hyperactivity disorder 2009-2011: focus on clinical efficacy and safety. $J$ Psychopharmacol 2014;28:204-11.

16 Vitiello B, Riddle MA, Greenhill LL, March JS, Levine J, Schachar RJ, et al. How can we improve the assessment of safety in child and adolescent psychopharmacology? J Am Acad Child Adolesc Psychiatry 2003:42:634-41.

17 Rawlins M. De testimonio: on the evidence for decisions about the use of therapeutic interventions. Lancet 2008;372:2152-61.

18 Singh S, Loke YK. Drug safety assessment in clinical trials: methodological challenges and opportunities. Trials 2012:13:138.

19 Langley K, Fowler T, Ford T, Thapar AK, van den Bree M, Harold G, et al. Adolescent clinical outcomes for young people with attention-deficit hyperactivity disorder. $\mathrm{Br} \mathrm{J}$ Psychiatry 2010;196:235-40.

20 Allison PD. Fixed effects regression methods for longitudinal data using SAS. SAS Institute, 2005
21 Wettermark B, Hammar N, Fored CM, Leimanis A, Otterblad Olausson P, Bergman U, et al. The new Swedish prescribed drug register-opportunities for pharmacoepidemiological research and experience from the first six months. Pharmacoepidemiol Drug Saf 2007:16:726-35

22 Lichtenstein P, Halldner L, Zetterqvist J, Sjolander A, Serlachius E, Fazel S, et al. Medication for attention deficit-hyperactivity disorder and criminality. N Engl J Med 2012;367:2006-14

23 Chang Z, Lichtenstein P, D'Onofrio BM, Sjolander A, Larsson H. Serious transport accidents in adults with attention-deficit/hyperactivity disorder and the effect of medication: a population-based study. JAMA Psychiatry 2014:71:319-25.

24 De Faire U, Friberg L, Lorich U, Lundman T. A validation of cause-of-death certification in 1,156 deaths. Acta Med Scand 1976;200:223-8.

25 Tidemalm D, Langstrom N, Lichtenstein P, Runeson B. Risk of suicide after suicide attempt according to coexisting psychiatric disorder: Swedish cohort study with long term follow-up. BMJ 2008;337:a2205.

26 Faraone SV, Glatt SJ. A comparison of the efficacy of medications for adult attention-deficit/hyperactivity disorder using meta-analysis of effect sizes. J Clin Psychiatry 2010;71:754-63.

27 Mann JJ, Arango VA, Avenevoli S, Brent DA, Champagne FA, Clayton P, et al. Candidate endophenotypes for genetic studies of suicidal behavior. Biol Psychiatry 2009;65:556-63.

28 Biederman J, Mick E, Faraone SV. Age-dependent decline of symptoms of attention deficit hyperactivity disorder: impact of remission definition and symptom type. Am J Psychiatry 2000;157:816-8.

29 McGough JJ, Smalley SL, McCracken JT, Yang M, Del'Homme M, Lynn DE, et al. Psychiatric comorbidity in adult attention deficit hyperactivity disorder: findings from multiplex families. Am J Psychiatry 2005;162:1621-7.

30 Sokero TP, Melartin TK, Rytsala HJ, Leskela US, Lestela-Mielonen PS, Isometsa ET. Prospective study of risk factors for attempted suicide among patients with DSM-IV majo depressive disorder. Br J Psychiatry 2005;186:314-8.

31 Food and Drug Administration. Summary minutes of the September 13-14, 2004 Center for Drug Evaluation and Research Pharmachopharmacologic Drugs Advisory Committee and the FDA Pediatric Advisory Committee. FDA, 2005.

32 Asherson $\mathrm{P}$, Adamou M, Bolea B, Muller U, Morua SD, Pitts M, et al. Is ADHD a valid diagnosis in adults? Yes. BMJ 2010;340:c549.

33 Moncrieff J, Timimi S. Is ADHD a valid diagnosis in adults? No. BMJ 2010;340:c547.

34 Faraone SV, Biederman J, Mick E. The age-dependent decline of attention deficit hyperactivity disorder: a meta-analysis of follow-up studies. Psychol Med 2006;36:159-65.

Accepted: 27 May 2014

\section{Cite this as: BMJ 2014;348:g3769}

This is an Open Access article distributed in accordance with the Creative Commons Attribution Non Commercial (CC BY-NC 3.0) license, which permits others to distribute, remix, adapt, build upon this work non-commercially, and license their derivative works on different terms, provided the original work is properly cited and the use is non-commercial. See: http://creativecommons.org/licenses/by-nc/3.0/. 


\section{Tables}

Table 1| Baseline characteristics of attention-deficit/hyperactivity disorder patients with and without suicide related events. Values are numbers (percentages) unless stated otherwise

\begin{tabular}{lcc} 
Characteristic & At least one suicide related event (n=3263) & No suicide related events (n=34 673) \\
Female sex & $1579(48.4)$ & $10968(31.6)$ \\
\hline Median (interquartile range) age, years & $21(16-29)$ & $17(13-27)$ \\
\hline Younger cohort born during 1982-96 & $2030(62.2)$ & $24609(71.0)$ \\
\hline Older cohort born during 1960-81 & $1232(37.8)$ & $10065(29.0)$ \\
\hline Use of drugs during follow-up: & $2197(67.3)$ & $21119(60.9)$ \\
\hline At least one prescription for stimulant & $654(20.0)$ & $5354(15.4)$ \\
\hline At least one prescription for non-stimulant & $2374(72.8)$ & $12855(37.1)$ \\
\hline At least one prescription for antidepressant & $1653(50.7)$ & $7580(21.9)$ \\
\hline Comorbid conditions before or during follow-up: & $510(15.6)$ & $1896(5.5)$ \\
\hline Depressive disorder & $290(8.9)$ & $1930(5.6)$ \\
\hline Bipolar affective disorder & $1540(47.2)$ & $4595(13.3)$ \\
\hline Conduct disorder & $625(19.2)$ & $1191(3.4)$ \\
\hline Drug abuse & &
\end{tabular}


Table 2| Baseline characteristics of attention-deficit/hyperactivity patients according to treatment patterns. Values are numbers (percentages) unless stated otherwise

\begin{tabular}{|c|c|c|c|}
\hline Characteristic & No treatment $(n=14485)$ & Intermittent treatment $(n=21$ 174) & Continuous treatment $(n=2277)$ \\
\hline Female sex & $4450(30.7)$ & $7531(35.6)$ & $566(24.9)$ \\
\hline Median (interquartile range) age, years & $19(15-26)$ & $17(13-28)$ & $14(12-20)$ \\
\hline Older cohort born during 1960-81 & $4244(29.3)$ & $6626(31.3)$ & $427(18.8)$ \\
\hline At least one suicide related event & $1059(7.3)$ & $2097(9.9)$ & $107(4.7)$ \\
\hline At least one stimulant treatment period & - & $19814(93.6)$ & $2233(98.1)$ \\
\hline At least one non-stimulant treatment period & - & $4896(23.1)$ & $486(21.3)$ \\
\hline At least one prescription for antidepressant & $5094(35.2)$ & $9430(44.5)$ & $705(31.0)$ \\
\hline \multicolumn{4}{|l|}{ Comorbid conditions before or during follow-up: } \\
\hline Drug abuse & $2116(14.6)$ & $3854(18.2)$ & $165(7.3)$ \\
\hline Borderline personality disorder & $619(4.3)$ & $1137(5.4)$ & $60(2.6)$ \\
\hline
\end{tabular}


Table 3| Hazard ratios for suicide related events during attention-deficit/hyperactivity disorder drug treatment periods, compared with non-treatment periods (2006-09)

\begin{tabular}{|c|c|c|c|c|c|c|}
\hline \multirow[b]{2}{*}{ Sex } & \multicolumn{3}{|c|}{ Population level } & \multicolumn{3}{|c|}{ Within patient level } \\
\hline & No of patients & $\begin{array}{c}\text { No of suicide related } \\
\text { events }\end{array}$ & Hazard ratio $(95 \% \mathrm{Cl})$ & No of patients & $\begin{array}{l}\text { No of suicide related } \\
\text { events }\end{array}$ & Hazard ratio $(95 \% \mathrm{Cl})$ \\
\hline Male & 25389 & 2903 & $1.32(1.17 \text { to } 1.48)^{*}$ & 13643 & 1806 & $0.79(0.64$ to 0.98$) \dagger$ \\
\hline Female & 12547 & 4116 & $1.34(1.17 \text { to } 1.54)^{*}$ & 7531 & 2809 & $0.88(0.76$ to 1.02$) \dagger$ \\
\hline Total & 37936 & 7019 & 1.31 (1.19 to 1.44$) \ddagger$ & 21174 & 4615 & $0.89(0.79$ to 1.00$) \dagger$ \\
\hline
\end{tabular}

*Adjusted for categorical age.

†Adjusted for categorical age, previous number of treatment switches, and previous number of suicide attempts.

$\ddagger$ Adjusted for sex and categorical age. 
Table 4| Sensitivity analyses for association between use of attention-deficit/hyperactivity disorder drugs and rate of suicide related events

\begin{tabular}{|c|c|c|c|c|c|c|}
\hline \multirow[b]{2}{*}{ Analysis } & \multicolumn{3}{|c|}{ Population level $^{*}$} & \multicolumn{3}{|c|}{ Within patient level† } \\
\hline & No of patients & $\begin{array}{l}\text { No of suicide } \\
\text { related events }\end{array}$ & Hazard ratio $(95 \% \mathrm{Cl})$ & No of patients & $\begin{array}{l}\text { No of suicide } \\
\text { related events }\end{array}$ & Hazard ratio $(95 \% \mathrm{Cl})$ \\
\hline $\begin{array}{l}\text { Treatment ended } 30 \text { days after last } \\
\text { prescription }\end{array}$ & 37936 & 7019 & 1.39 (1.27 to 1.53$)$ & 21125 & 4599 & 0.96 (0.86 to 1.08$)$ \\
\hline $\begin{array}{l}\text { Treatment status defined by } 3 \\
\text { month cut-off }\end{array}$ & 37936 & 7019 & $1.40(1.26$ to 1.56$)$ & 22883 & 4718 & $0.90(0.80$ to 1.01$)$ \\
\hline Stimulant users & 16460 & 3322 & $1.02(0.90$ to 1.16$)$ & 14757 & 3191 & $0.81(0.70$ to 0.94$)$ \\
\hline Non-stimulant/mixed users & 6992 & 1493 & 1.49 (1.27 to 1.76$)$ & 6417 & 1424 & $0.96(0.77$ to 1.20$)$ \\
\hline $\begin{array}{l}\text { Stimulant treatment periods } v \\
\text { non-treatment periods }\end{array}$ & - & - & 1.45 (1.22 to 1.73$)$ & - & - & $0.93(0.72$ to 1.20$)$ \\
\hline $\begin{array}{l}\text { Non-stimulant treatment period } v \\
\text { non-treatment periods }\end{array}$ & - & - & $1.48(1.17$ to 1.88$)$ & - & - & $0.96(0.72$ to 1.30$)$ \\
\hline $\begin{array}{l}\text { Younger cohort born during 1982-96 } \\
\text { (baseline age 10-24 years) }\end{array}$ & 26639 & 4214 & $1.17(1.05$ to 1.31$)$ & 14548 & 2699 & $0.92(0.79$ to 1.07$)$ \\
\hline $\begin{array}{l}\text { Older cohort born during } 1960-81 \\
\text { (baseline age } 25-46 \text { years) }\end{array}$ & 11297 & 2805 & 1.39 (1.20 to 1.61$)$ & 6626 & 1916 & 0.82 (0.68 to 0.99$)$ \\
\hline $\begin{array}{l}\text { Patients without comorbid } \\
\text { conditions }\end{array}$ & 22555 & 946 & 1.24 (1.03 to 1.48$)$ & 11873 & 587 & $1.08(0.69$ to 1.70$)$ \\
\hline $\begin{array}{l}\text { Patients with lifetime depressive } \\
\text { disorder }\end{array}$ & 9233 & 4208 & $1.28(1.13$ to 1.43$)$ & 5584 & 2796 & $0.78(0.68$ to 0.91$)$ \\
\hline $\begin{array}{l}\text { After adjustment for use of } \\
\text { antidepressants }\end{array}$ & - & - & 1.14 (1.02 to 1.29$)$ & - & - & 0.77 (0.66 to 0.89$)$ \\
\hline
\end{tabular}

*Adjusted for sex and categorical age.

†Adjusted for categorical age, previous number of treatment switches, and previous number of suicide attempts. 